INTERNATIONAL DESIGN CONFERENCE - DESIGN 2018

https://doi.org/10.21278/idc.2018.0142

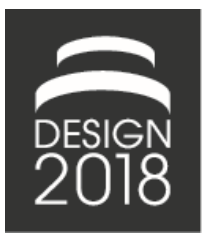

\title{
USING THE NEW WORKING SPACE MODEL FOR THE DEVELOPMENT OF HYGIENIC PRODUCTS
}

\author{
J.-P. Beetz, P. D. Schlemmer, H. Kloberdanz and E. Kirchner
}

\begin{abstract}
Hygienic design is of fundamental importance in the development of food processing machines. A hygiene appropriate design leads to lower maintenance work for machine operators and to clean food free of contamination. Designers have access to plenty of guidelines that support the embodiment design and detailing of specific equipment. The Working Space Model is a suitable adaptation of the wellknown and established Contact \& Channel Model, which aids designers in systematically considering hygiene-relevant requirements during the conceptual design.
\end{abstract}

Keywords: hygienic design, conceptual design, design for $x(D f X)$, early design phase

\section{Introduction: Hygienic Design}

Hygienic Design is a key discipline in the development of food-processing machinery with the aim of reducing risks of contamination. Hygienic Design guidelines support designers during the embodiment design or detailing by giving exemplary illustrations of specific components about possibilities to minimise the risk of contamination. They mainly consist of figurative illustrations like examples that show design suggestions for specific components (Beetz et al., 2017).

However, they do not fully exploit the potentials of Hygienic Design since these guidelines mainly focus late design phases. This paper presents an approach to extend Hygienic Design in order to support designers developing new and innovative hygienic products especially during early design phases.

The established Contact \& Channel Model represents a mostly suitable approach for the early consideration of functional and design requirements. However, hygienic design relevant issues like leakage flows between components, dead spaces or similar topics, are not subject of the Contact \& Channel Model so far. Therefore, this paper presents an extension, the so-called "Working Space Model". The goal is to increase possibilities for designers of influencing hygiene concerning decisions. In order to achieve this goal, this paper addresses following research questions:

- How can products be described in order to derive Hygienic Design characteristics?

- How can Hygienic Design characteristics be considered in early phases of the development?

- To what extent does the product model support the synthesis of new hygienic products?

\section{Motivation: Food processing and the need for Hygienic Design}

Awareness of the consequences of hygiene-inappropriate design arises when it comes to personal diet. A particular example of food processing is highlighted below: the production of pralines. In 2016, German companies have produced 1.1 million tons of chocolate products, which corresponds to a total value of 5.3 billion euro. Of this amount, 325,500 tons are filled chocolate bars and about 144,000 tons pralines (BDSI, 2017). For the production of filled chocolate products, as in Figure 1, various production 
technologies exist that have a different complexity and differ in the chocolate product quality (Ziegleder et al., 2005):

- Centrifugal casting technology,

- Cold stamping technology,

- One-Shot technology.
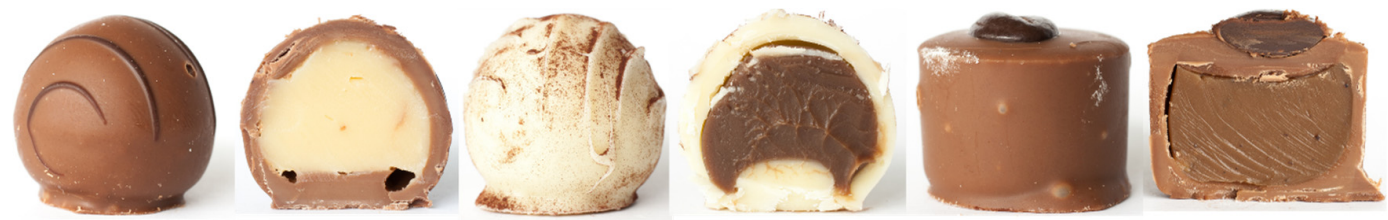

Figure 1. Chocolate products with various fillings (pralines), own illustration

The production of chocolate products with the centrifugal casting technology and the cold stamping technology consists of several steps. Both technologies starts with the production of the chocolate shell. Subsequently, the shell is filled with the desired compound. Each process step requires a separate machine. The One-Shot technology allows pouring filled chocolate products in a single process step. Based on the simultaneous casting of the shell and the filling compounds, it is possible to achieve the following immediately apparent advantages, Figure 2:

- One-Shot machines are very compact,

- Production and operating costs of the machine are lower in comparison with other production technologies.

Existing dosing pumps for the production of chocolate products currently have a major problem, since they require a considerable cleaning effort in terms of time and assembly complexity. The cleaning staff has to disassemble, clean and reassemble many (sometimes very complex) components. This carries the risk of errors in assembly or poorly cleaned components.

The following exemplary development of a new, hygienic pump for a chocolate depositor at the end of the paper demonstrates the advantages of the Working Space Model for designers.

\section{Functional and processual analysis of food processing machines}

Due to integration of different processes into the One-Shot technology, intermediate cooling and further processing of chocolate is no longer necessary (Haendler, 2008). This eliminates the need for additional machines for these processes and minimises space requirements, as well as investment costs, see Figure 2a. By reducing the amount of process steps, the One-Shot technology is especially time and cost-saving compared to the other manufacturing technologies.

The dosage of all necessary compounds for the praline originates from the respective compound storages. With the help of separate piston pumps, all dosed praline compounds pass through a joint, concentric ring nozzle into a mould, Figure $2 \mathrm{~b}$. The timed sequence of the dosing is of great importance for a precise shape of the praline, Figure 2c. The casting of the shell compounds takes place first, so that the shell of the praline develops. Shortly after, the injection of the filling proceeds simultaneously. Finally, the shell compound completes the praline casting process. During the casting process, the nozzle moves steadily upwards, which improves the praline shape. The production time of a praline with the One-Shot technology is about 3-5 seconds per praline compared to up to 100 times longer with other industrial technologies (Haendler, 2008).

Nevertheless, in addition to the mere functionality of the machine, the fundamental challenge for designing food-processing machines is also the fulfilment of hygiene requirements. An advanced and timesaving production technology is only successful if it does not entail additional cleaning costs for the machine operator and if it reduces the risk of contamination. A machine with a consistent Hygienic Design can support the enthusiasm of machine operators (less maintenance work) and leads to products free of contamination.

The dosing pump has a high level of technical difficulty from the point of view of designers and great importance for the customer. Therefore, it represents the key component of the chocolate dosing 
machine. Dosing pumps are a special type of displacement pumps. There is a distinction between constant volume pumps and variable displacement pump (Vetter, 1995). The task of dosing pumps is to extrude a defined amount of fluid. They deliberately do not generate a continuous volume flow.

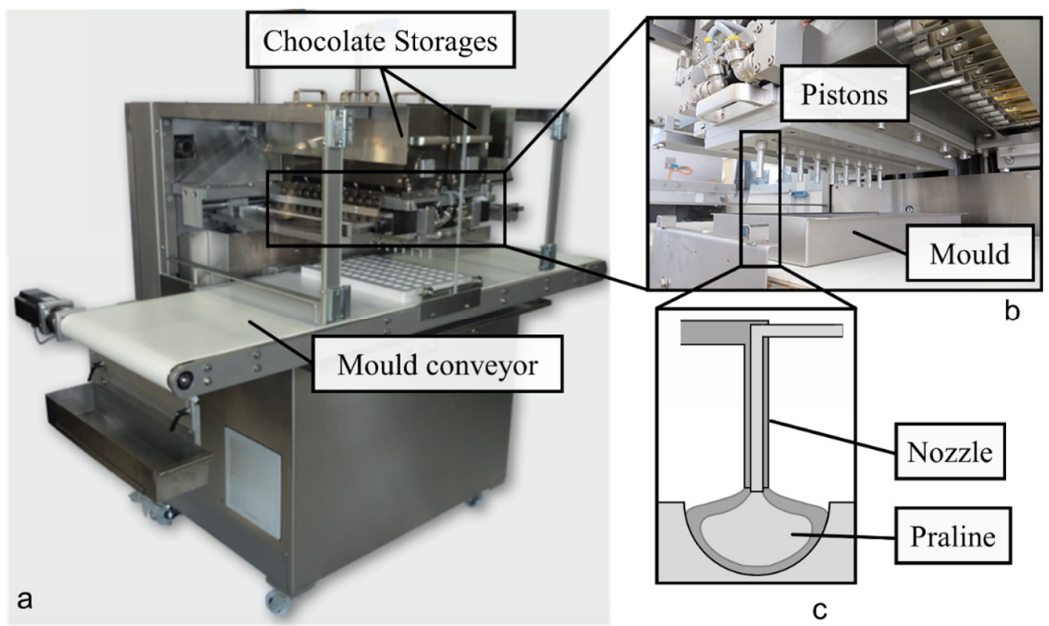

Figure 2. a: Chocolate depositor machine referring to (AWEMA, 2017), b: Detailed view on pistons, c: Schematic sketch of the One-Shot-Process

Currently, almost every dosing pump in praline manufacturing is a piston pump. They allow a pulsationfree extrusion. The essential components of piston pumps are the piston and the cylinder, as well as valves that are required to separate the inlet from the outlet. By connecting the inlet to the dosing chamber and the movement of the piston, the dosing volume forms and fills with chocolate, Figure 3. The covered distance by the piston determines different amounts of chocolate. To squeeze out the dosage volume, the valve closes the connection between the inlet and the dosing chamber and moves to a position connecting it to the outlet.

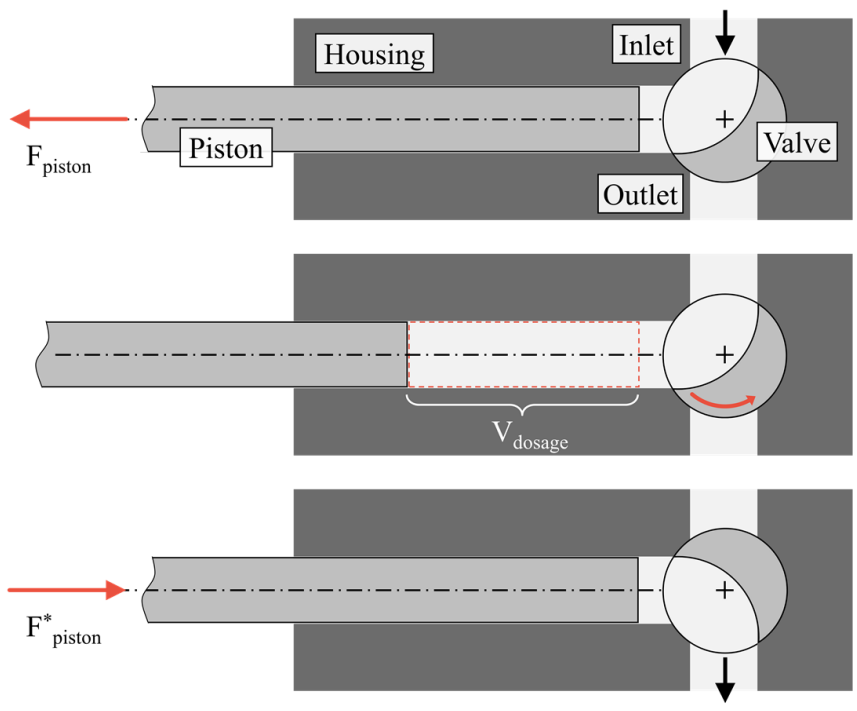

Figure 3. Working principle of the dosing piston pumps

This analysis of an existing pump already shows that there are different possibilities for changes. For example the implementation of a different working body of the piston, the modification of the working direction of the piston (which does not necessarily have to be translational) or the variation of the valve design and/or the working direction. A modification of the dosing pump concept may lead to an improvement in terms of hygiene by reducing the risk of contamination and the necessary cleaning effort. 


\subsection{Limitations of analysing with the help of the Contact \& Channel Model}

Matthiesen (2002) describes the "Contact \& Channel Model" (C\&CM) as a design approach that depicts the function and shape of a product in a common model. The model essentially bases on so-called Working Surface Pairs (WSP) as well as on Channel and Support Structures (CSS). Only the interactions of two Working Surfaces as a Working Surface Pair is responsible for the realisation of technical functions in a mechanically oriented framework. Furthermore, Matthiesen (2002) postulates that a function needs at least two Working Surface Pairs and a connecting Channel and Support Structure. Moreover, Matthiesen claims that each function fulfilling system consists of Working Surface Pairs and Channel \& Support Structures that may occur in any number, arrangement and form.

Figure 4 shows an example application of the C\&CM for the analysis of the dosing piston pump. On the one hand, it is apparent, that the fluid (chocolate in this case) needs a support from all directions inside the pump, which realises the guide of the fluid (WSP 3, WSP 4, WSP 5, WSP 8 and WSP 9). For the extrusion, the Working Surface of WSP 2 exerts a pressure on the fluid. The other way round, by moving the working surface of the piston outward, partial negative pressure results, which leads to a suction of the fluid into the chamber (not illustrated in Figure 4). By means of the C\&CM-analysis, a description of the working principle of the system in a comprehensible way is possible; it facilitates the reconsideration of existing arrangements.

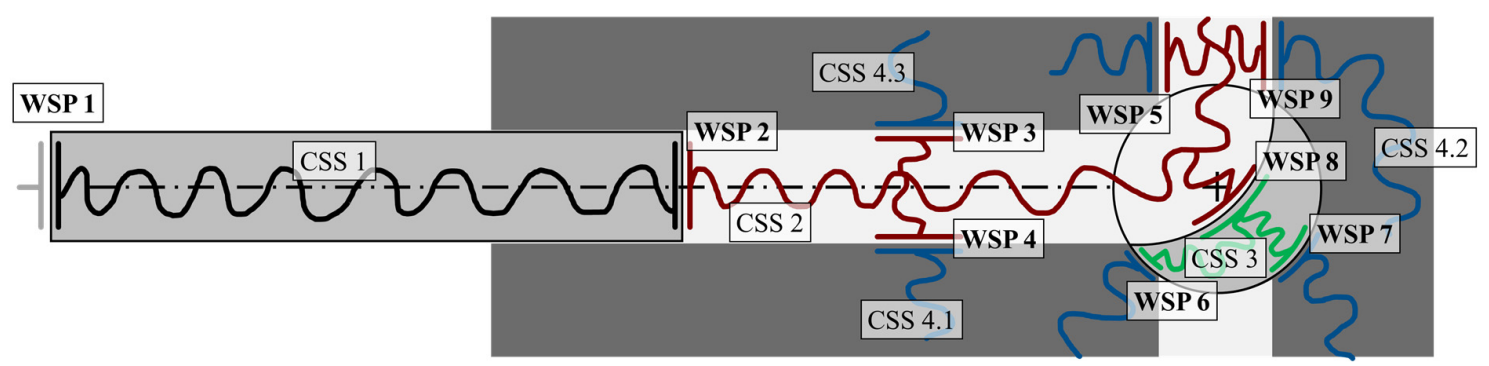

Figure 4. Contact \& Channel-Model Analysis

The prevention of potential sources of contamination represents an important basis of Hygienic Design. It is not trivial for designers to illustrate hygienic design relevant issues using the C\&CM.

The consideration of these issues is an important prerequisite with regard of a holistic implementation of hygienic design requirements. An adaptation and expansion of the C\&CM is necessary, in order to take Hygienic Design relevant information as early as possible into account.

\subsection{The context of processes in Hygienic Design in: The Detailed Process Model}

Hygienic Design is of vital importance whenever food processed and changes its state (Beetz et al., 2017). The example of the piston pump is of relevance to hygienic design because it transforms the chocolate from an undosed state ("initial state") to a dosed state ("final state"), Figure 5. For the transformation or for the progression of the process a "working factor" is necessary that affects the food. Design elements of the so-called "food area" (the term derives from hygienic design guidelines) provide the working factor for the process (Beetz and Kloberdanz, 2016). In the piston pump example, the front surface of the piston and all surfaces that prevent the uncontrolled discharge of food represent design elements. All these design elements that are indispensable for processing the food ("process-relevant design elements") belong to the food area. The most rigorous hygiene-requirements apply to this area. In addition to the working factor, which represents an intended relation, there are also unwanted interactions between design elements and food. Depending on the arrangement, position or other properties of design elements, food may, adhere to the design elements or reach areas that are not intended for food contact ("non-food area"), for example drive and gear units or bearings. In summary, this means that the food area is exclusively utilisable for food contact, whereas the non-food area must constantly remain free from food. Furthermore, there is a risk of food getting into contact with the environment. Thereby the risk of contamination increases. The designer has ensure that these unwanted interactions do not occur, Figure 5 (Beetz et al., 2017). 


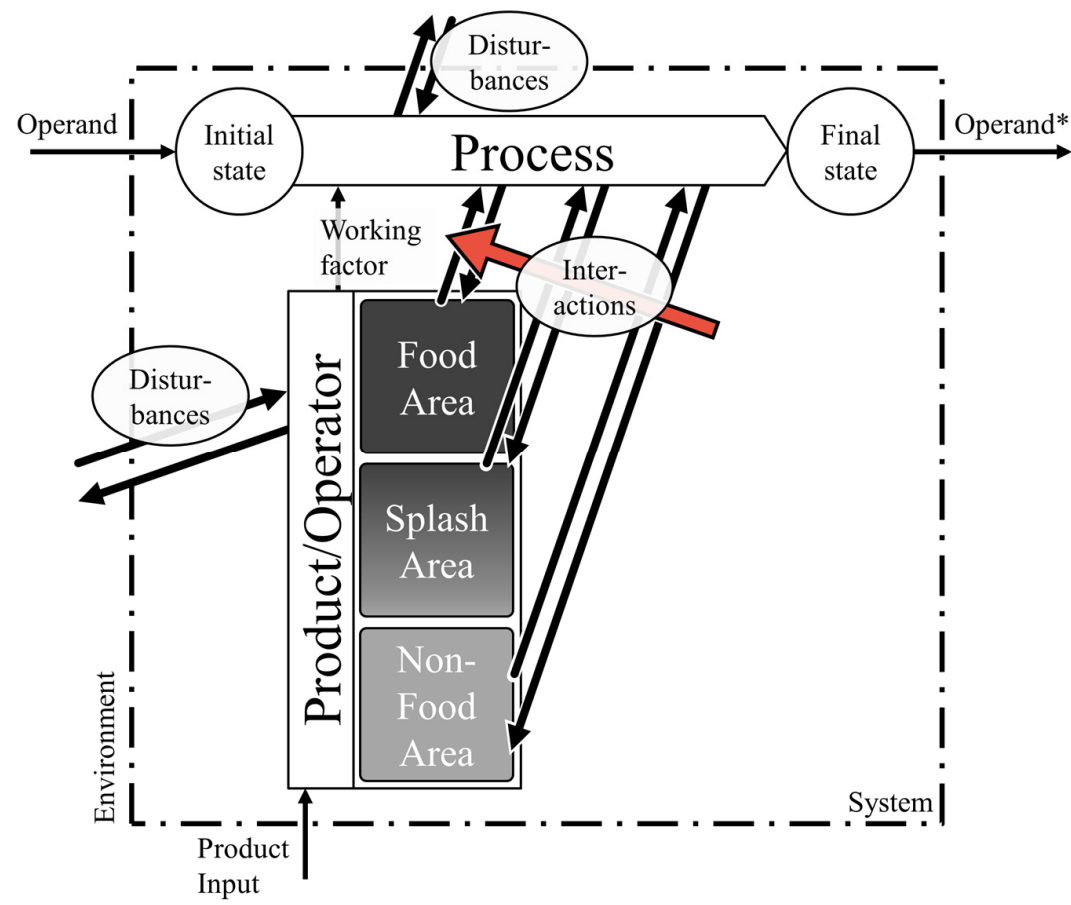

Figure 5. Detailed Process Modell

\subsection{Early consideration of Hygienic Design using the Working Space Model}

When it comes to the development of food processing machines, wherein the food is in a liquid, pasty or powdery state, it is advisable using so-called "Working Spaces". The implementation of this term is necessary for the proper distinction between solid components of the machine, excipients and auxiliary gases or liquids, as well as the processed food. A Working Space is a volume bounded by working surfaces or boundary surfaces of working bodies or adjacent Working Spaces. Working Spaces also have CSS and enveloping surfaces that are in contact with other surfaces as WSPs or just as boundary surfaces. Furthermore, each Working Space fulfils a function. Figure 6 illustrates the idea of assigning functions to Working Spaces and possible hygiene-risks. The analysed piston pump from the previous chapters consists of:

- Working Space 1 (WS 1), inlet for inflowing food,

- Exchange room (WS 2), which ensures that the right rooms connect at the right time,

- Antechamber (WS 3) that guides food between WS 2 and WS 4,

- Dosage (WS 4), which is meaningful for the overall function of the machine. The larger the volume difference between top and bottom dead centre, the larger the dosed volume. Beyond that, the dosed food volume is extruded with the help of the Working Surface of the piston and its Working Movement,

- Outlet (WS 5) that enables and leads the dosed food to get out of the system.

Besides the functional consideration of the Working Spaces, the analysis with the help of this model also allows the inclusion of requirements, which is necessary for Hygienic Design. The WS model supports the determination of contamination risks not only on Working Surfaces itself, but also on unwanted connections between two WSs. Figure 6 illustrates that WS 4 has an unsealed connection to the environment, which can lead to leakage. The other way round, bacteria might also intrude into the system through the unsealed connection of WS 6 and the environment. The model may also illustrate unintentional dead spaces, such as the connection between WS 1 and WS 5 via the valve. The prevention of these unintended dead spaces or unwanted connections to other areas form an important base in the early consideration of Hygienic Design. 


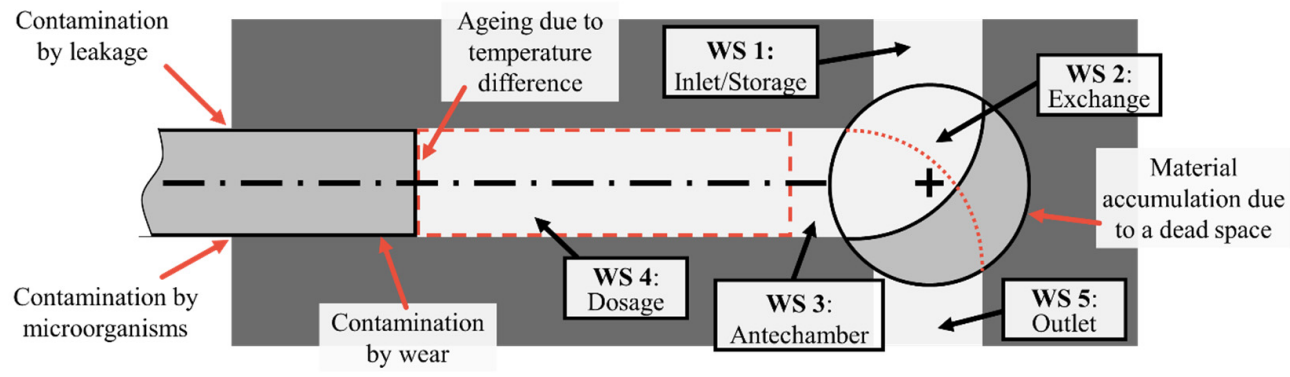

Figure 6. Working Spaces with hygiene-risks

In order to analyse the system systematic way, which does not contain information about the detailed design, a different presentation is necessary, Figure 7. Figure 7a shows the system during the dosing process, Figure $7 \mathrm{~b}$ shows it during the squeezing process of the food with the help of the developed Working Space Model. The boxes illustrate the WSs from Figure 6 in an orderly manner. It is not only topological arrangement of the rooms, but also according to their geometric height. Connections between WSs depend on the respective process step. The arrows represent the food flow. For example, in Figure 7a, WS 5 (outlet) has no connection to WS 2 (exchange) during the dosing process. This is indispensable because during dosing, the food should originate from the storage. Therefore, there must be no connection between the inlet and the metering during the squeezing process. Figure $7 \mathrm{c}$ illustrates the temporal projection of all connections within the system. Dashed connections exist only intermittently, whereas solid connection exist continuously. As explained earlier, the illustration only shows spaces that do not contain solid material. This is useful for the development of hygiene-relevant products, as unwanted material flows or connections are detectable. Hygienic Design aims primarily on the analysis of the material/food flow and a further realisation of an adequate design based on an appropriate concept.
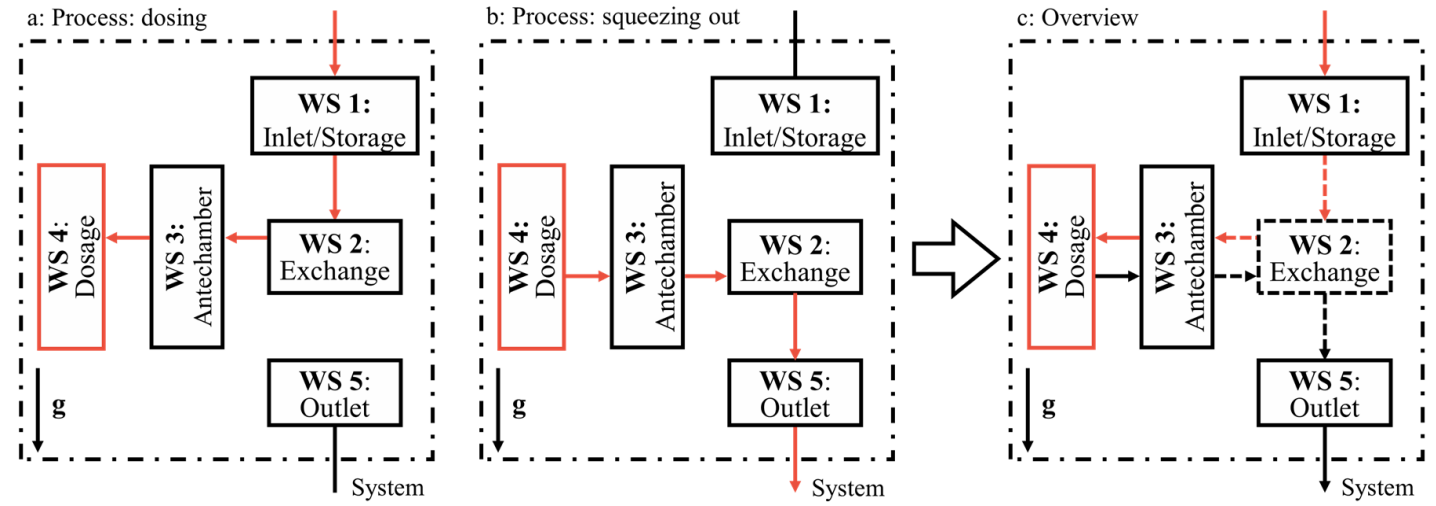

Figure 7. Working Space Model (a: during the dosing process, b: during the squeezing process, c: time-independent overview)

The new Working Space Model relates directly to the C\&CM. It is a specialisation of well-known established models with the focus on hygienic products. It is necessary to recognise the connection to the "classical" product development. It is due to the fact that the boundaries of the WSs - the Working Surfaces - are in direct contact with other Working Surfaces of the machine. These, in turn, are part of a system that, of course, follows the rules and methods of the methodical product development. The extension of the C\&CM by the aspect of WSs is therefore necessary through the processing of the food.

\subsection{Derivable Hygienic Design characteristics from the Working Space analysis}

With the help of the Working Space Model, it is possible to display and analyse complex systems in an understandable and clear way. In the analysis, the Working Space Model focuses on the function of spaces filled with, for example, food. The Working Space Model simplifies deriving design requirements, e.g. according to the arrangement of WSs. The following Working Space Model characteristics are of special interest in the field of Hygienic Design. 
Functional and design analysis of the system:

The Working Space Model facilitates designers considering functions of all essential elements of the system. Thus, it is also possible to identify spaces that are insignificant for the functionality. The elimination of these spaces therefore leads to a possible simplification and may lead to a hygienic improvement. Simplified designs, which do not have redundant and complex components, may lead to products that are hygienic design compliant. Spaces that are insignificant for the functionality represent possible dead volumes where food might spoil. They also increase the cleaning effort.

Furthermore, it becomes apparent, which adjacent surfaces and which surface pairs are necessary for the realisation of the respective function, Figure 8a. These necessary elements are Working Surfaces and Working Surface Pairs, Section 2.1.

\section{Contamination risks:}

The Working Space Model supports designers identifying unwanted but existing connections between the WSs and the surrounding air or other spaces of the environment. The elimination of unwanted connections is of great importance in Hygienic Design. Unwanted connections carry the risk of food spoiling into dead spaces or unwanted substances (such as microorganisms or dirt) intruding into the system, especially into the food area. The model therefore supports designers to uncover the unwanted connections (red lines of Figure $8 \mathrm{~b}$ ) during the analysis. The model does not point out these unwanted connections by itself, but helps designers during an analysis.

\section{Variation of the topology:}

The topology, i.e. the arrangement and the connection of Working Spaces, plays a key role in Hygienic Design. For example, the design must be in a way that food can always flow out of the system independently (EHEDG, 2004). As a result, this constraint prevents the formation of puddles, which are potential sources of contamination. A further example of the importance of the topology in Hygienic Design concerns the cleanability. The machine design must prevent the forming of detergents puddles in a way that they drain off independently. The Working Space Model supports designers illustrating the Working Space topology, Figure 8c.

\section{Entry point for a Failure Mode and Effects Analysis (FMEA):}

The functional character of the Working Spaces and the connections of the spaces can support development teams on the execution of a FMEA. Especially the consideration of possible connections between Working Spaces of the food area and the environment, and between Working Spaces of the food area and the adjacent system (splash area and non-food area) are expedient. If, for example, seals that are supposed to prevent the unwanted connection fail, hygienic risks may arise. For this purpose, designers must define appropriate measures.
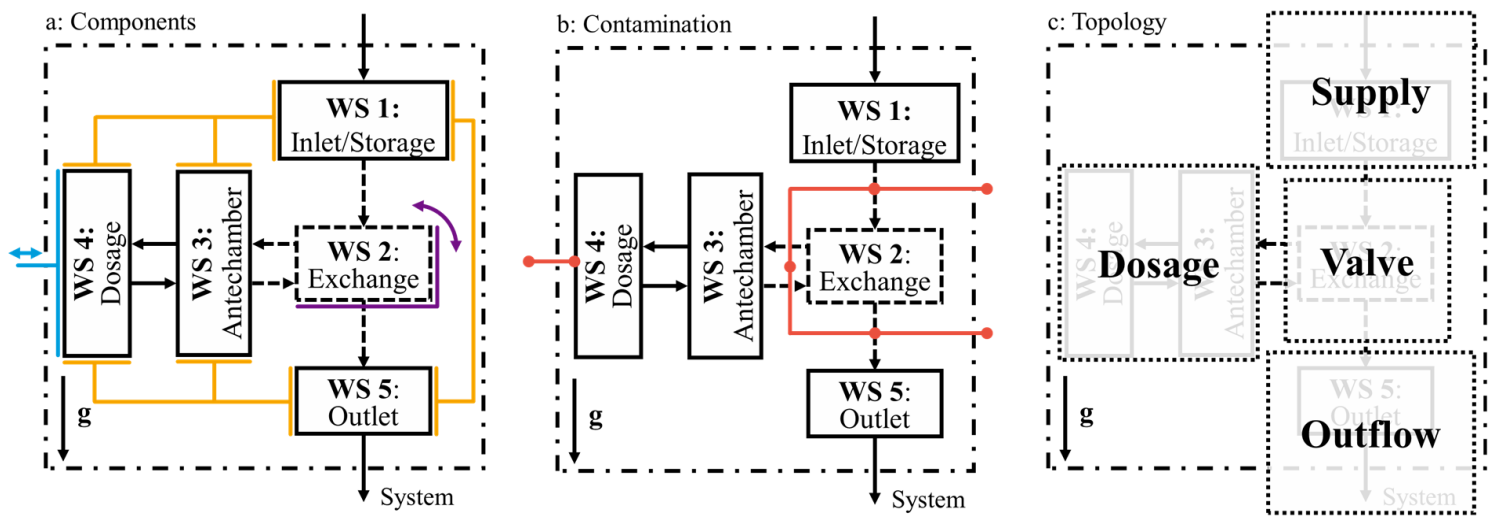

Figure 8. Derivable characteristics from the Working Space Model (a: Connected components, b: Illustration of potential contamination sources, c: Topology of the system) 


\section{Hygiene-appropriate redesign of a chocolate depositor}

The following section demonstrates the potentials of the Working Space Model as an effective support for the development of new hygiene-appropriate products. This example presents the development of a new dosing pump for processing chocolate. Starting point of the development is a dosing machine, Figure 9. A collective drive enables the simultaneous movement of all dosing pumps (not shown in Figure 9). The aim of the development is to minimise the risks of contamination while simplifying cleaning at the same time. The new machine should base on the same working principle (volumetric dosing) as the initial machine.

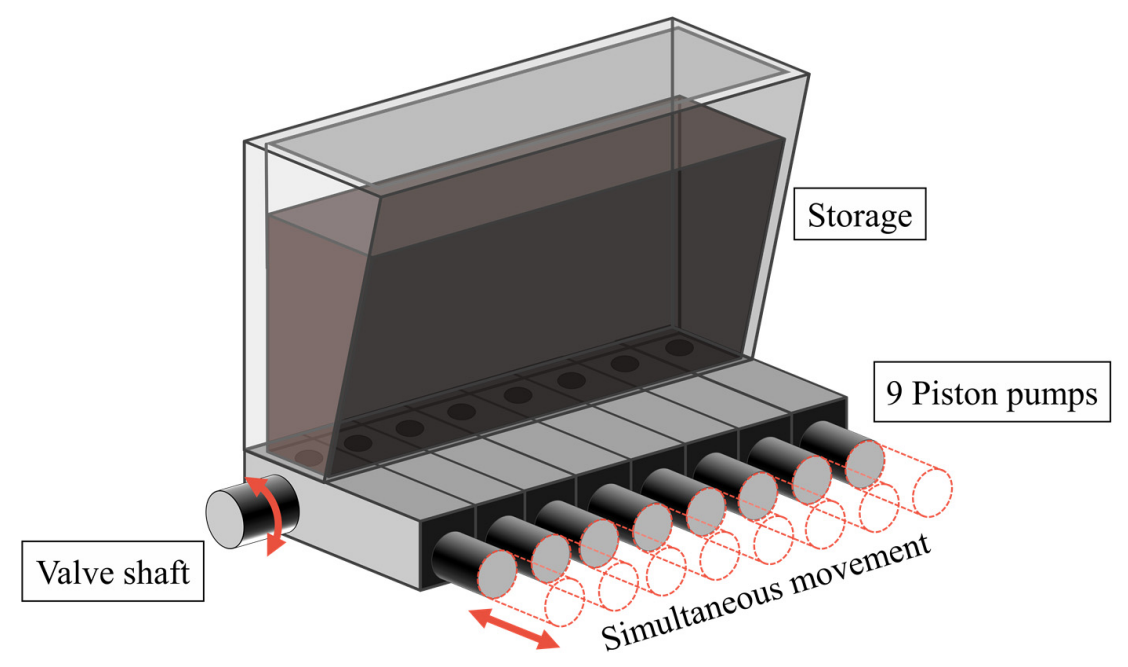

Figure 9. Initial dosing pump machine with nine parallel piston pumps

\subsection{Working Space analysis of an existing piston depositor}

The initial machine design consists of nine parallel piston pumps whose structure is similar to that of Figure 3. On the one hand, the following aims on determining the functions of the Working Spaces and detection of essential Working Surfaces, which are necessary for the realisation of the dosing process. On the other hand, the goal is to analyse contamination risks in order to capture systematically potential starting points for a hygienic improvement. Figure 11a shows the Working Space Model of the machine. Red lines indicate unwanted connections of Working Spaces and Working Space connections. They are focused in this investigation. They represent potential dead spaces or gaps in which food can accumulate or unwanted material can intrude into the system from the environment. The new concept should reduce the amount of unwanted connections.

- Unwanted connection 1: Connection between storage and ambient air. Putting on a cover lowers the risk of a contamination. This connection is not focus of this investigation.

- Unwanted connections 2.1 und 2.2: Connections between storage and inlet to the ambient air. This connection is visible in Figure 9 (between the storage and the nine piston housings). In the event of a leak, the food can unintentionally flow out in many places (via connection 2.1 to the side and via connection 2.2 to the bottom) and pollute the environment. Apart from that, there is a risk of intrusion of unwanted substances from the outside through these connections.

- Unwanted connections 3.1, 3.2, 3.3: Connection around exchange and ambient air. The working principle and the design of the valve shaft causes this connection. The rotation axis of the valve shaft is orthogonal to the longitudinal axis of the piston. Therefore, there is a further connection into the environment. The separation between all housing creates additional connections.

- Unwanted connection 4: Connection between dosage and ambient air. The stroke movement of the piston creates a direct connection between dosing chambers and ambient air. As a result, food gets to the environment and unwanted substances get into the food area. There is great potential 
for improvement as each piston has the same vulnerability. Therefore, a new concept offers great potentials for improvements in terms of hygienic design.

In summary, the initial dosing pump machine fulfils customer's expectations by allowing a precise dosing of the chocolate. Yet, many unwanted connections between the food area and ambient air exist and lead to a non-hygienic solution. In addition, cleaning personnel needs to disassemble all contaminated components in every cleaning cycle. Since the machine contains many components, disassembly causes high maintenance effort. A new pump concept therefore offers the potential for extensive improvements.

\subsection{New hygiene-appropriate design of the depositor}

Since the working principle should remain the same, the variation of the following properties is expedient (Pahl et al., 2007):

Working body/surfaces:

- Number (simple, multiple, ...)

- Behaviour (rigid, elastic, ...)

- Position (axial/radial/tangential, parallel/sequential, ...)

\section{Working motions:}

- Type (stationary/moving, translational/rotational, ...)

- Nature (uniform/non-uniform/oscillating, ...)

Systematically combining the varied properties produces different concept variants. The major hygiene weak point of the previous piston pump is the connection between the dosage chambers and the environment (see Figure 11a, red line no. 4). The conceptual design therefore focuses on variants where leakage flows do not directly get into the environment.

The goal is therefore to prevent the leakage flow either completely by a leak-free variant. Alternatively, a variant is conceivable, in which the leakage does not get into the environment but back into the food area. Figure 10 shows a new concept. Instead of a translational working motion, the motion is rotational. A counter-holder within the circumference saves the additional valve shaft.

The entire dosing pump unit consists of nine successive dosing chambers. Figure 10 shows a cross section of one chamber. Figure 11b illustrates the corresponding Working Space Model.

The new dosing pump concept achieves following hygiene-concerning improvements (see also Figure 11b):

- Nine dosage chambers with common shafts driven without a converter.

- Only the outer chambers are in contact with the environment. Seals can prevent leakage and intrusion of foreign matter. The entire dosage unit is encapsulated.

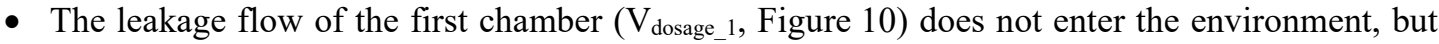
returns to the second chamber $\left(\mathrm{V}_{\text {dosage_}} 2\right)$, which ensures that the leakage is not hygiene-critical.

The conceptual design requires further concretisation in order to make accurate statements on quantitative improvements, e.g. assembly, cleaning or contamination.
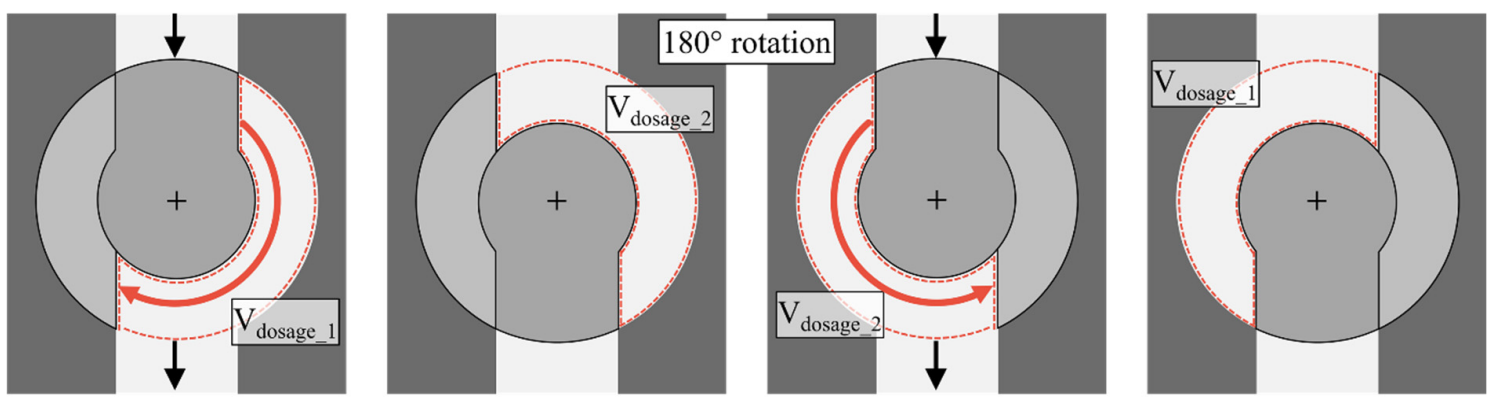

Figure 10. New dosing pump concept, section of one chamber 



Figure 11. a: Working Space Model applied on piston pump system b: Working Space Model of the redesign 


\subsection{Concretisation of the hygiene-appropriate redesign}

Conceivable variants embodiments differ by the amount of components, their arrangement, type of connections, etc. The variation of Channel and Support Structures represents the basis for the systematic development of embodiment variants. Figure 12a shows the underlying Design Structure Matrix. In particular, Working Surfaces, which either can have a connection or can be separated (Figure 12a, orange), are important. Figure $12 \mathrm{~b}$ shows the most promising embodiment design variant in terms of Hygienic Design.

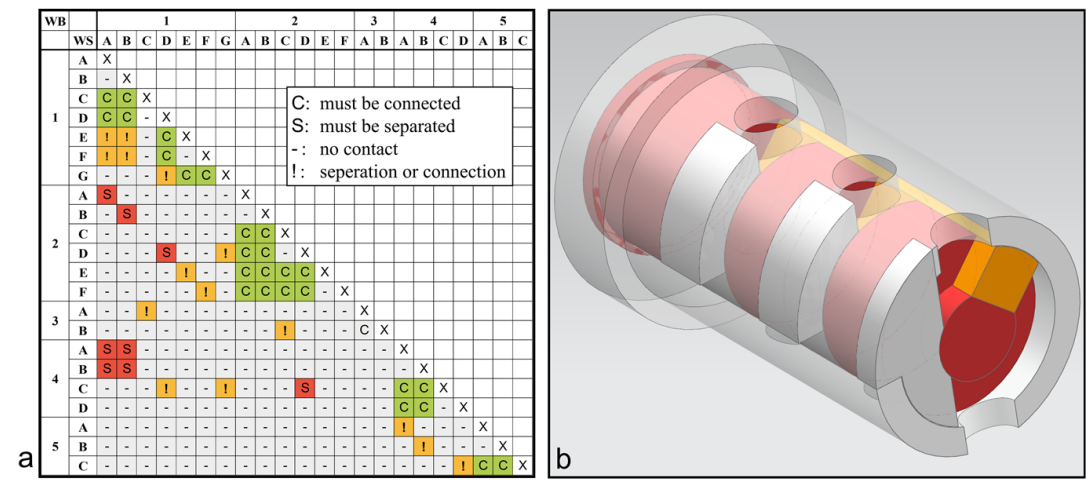

Figure 12. a: Design Structure Matrix of the concept, b: Concretisation of the concept

Figure 13 shows the final machine, with two dosing units and nine dosage chambers each. One indication of the system's vulnerability to contamination, represents the number of unwanted connections with the environment, see Section 2.4. According to Figures 10a and 10b, there is a different number of unwanted connections to the environment. The number of components and the number of steps required to disassemble the system constitute an indication of the cleaning effort, if cleaning of the machine is out of place. Table 1 shows the summed up numbers. (They refer to nine parallel dosing chambers.)

Table 1. Comparison of the dosing concepts

\begin{tabular}{lll}
\hline \hline Criterion & Piston pump & New dosing pump \\
\hline Number of unwanted connections to environment $^{\mathrm{a}}$ & $\sim 29$ & $\sim 6$ \\
Number of components $^{\mathrm{b}}$ & $\sim 22$ & $\sim 15$ \\
Number of assembly steps for cleaning $^{\mathrm{b}}$ & $\sim 33$ & $\sim 16$ \\
\hline \hline
\end{tabular}

${ }^{\mathrm{a}}$ Accurate number depends on the granularity of the analysis; ${ }^{\mathrm{b}}$ Screws and seals are not taken into account

The comparison illustrates the advantages of the new dosing pump. It has a lower number of unwanted connections, which is mainly because the entire dosing unit is encapsulated and only the outer ends need seals. From the customer's point of view, a significant advantage over the previous piston pump is that the number of components is also lower, so fewer parts have to undergo a cleaning process. In particular, there are fewer assembly/disassembly steps because not every chamber requires a single housing and a single piston.

\section{Conclusion}

The consideration of hygienic design in the field of food machinery plays an essential role in multiple ways. Operators of the machine get the possibility of an easy cleaning and the consumer of the food receives a contamination-free food product. So far, designers in the context of Hygienic Design only have documents that support late stages of product development. Usually, designers discover contamination risks after prototyping and testing. The designer has to eliminate these weaknesses thereafter. The newly developed Working Space Model, which represents a suitable adaptation of the Contact \& Channel Model, enables the early inclusion of hygiene-relevant requirements into the product development process. The model supports designers during functional and design analysis of systems, the identification of contamination risks in the form of unwanted connections and in the variation of the 
topology. The functional character of the Working Spaces also offers the opportunity to use the model as an entry point for an FMEA. This paper exemplifies the use of the Working Space Model as the basis of a Hygienic Design oriented analysis. Using the results of the analysis leads to a new and hygieneappropriate dosing pump. The results are enriched by elements of the Contact \& Channel model and other established methods, such as the systematic variation of working elements,

This new perspective in the field of Hygienic Design offers a large number of possibilities that support the systematic development of food processing machines.

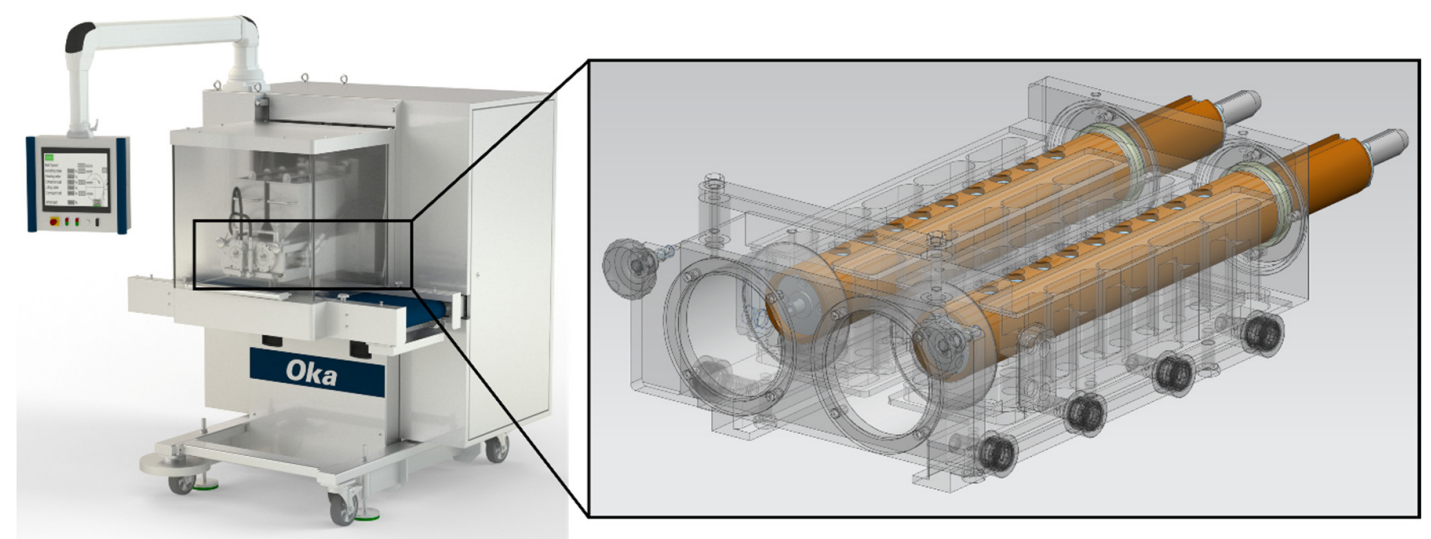

Figure 13. Realised concept of a new hygienic dosing pump system

\section{Acknowledgement}

Thank you to the German Federation of Industrial Research Associations - AiF - for funding this work.

\section{References}

AWEMA (2017), One-Shot and universal depositing machine. [online] AWEMA AG. Available at: http://www.awema.ch/data/AWEMA_UDM101_e.pdf (accessed 08.03.2018).

BDSI (2017), Facts \& Figures. Chocolate, Chocolate Products and Cocoa. [online] Association of the German Confectionery Industry. Available at: https://www.bdsi.de/en/facts-figures/chocolate-chocolate-products-andcocoa (accessed 08.03.2018).

Beetz, J.-P. and Kloberdanz, H. (2016), "Modellbasierte Anforderungsermittlung durch systematische Prozessanalyse als Basis des Hygienic Designs", Proceedings of DFX 2016 / the 27th Symposium Design for X, Jesteburg, Germany, October 5-6, 2016, Tutech Verlag, Hamburg, pp. 207-218.

Beetz, J.-P., Kloberdanz, H. and Kirchner, E. (2017), "New ways of hygienic design - A methodical approach", Proceedings of ICED '17 / the 21st International Conference on Engineering Design, Vol. 5, Vancouver, Canada, August 21-25, 2017, The Design Society, Glasgow, pp. 81-90.

EHEDG (2004), DOC8: Hygienic Equipment Design Criteria, 2nd ed. [online] European Hygienic Engineering and Design Group, Frankfurt, Germany. Available at: https://www.ehedg.org/guidelines

Haendler, H. (2008), “Triple-Shot - Impfgießen auf einem anderen Niveau”, Süsswaren, Vol. 53 No. 1, pp. 1819.

Matthiesen, S. (2002), Ein Beitrag zur Basisdefinition des Elementmodells "Wirkflächenpaare \& Leitstützstrukturen" zum Zusammenhang von Funktion und Gestalt technischer Systeme, PhD thesis, Universität Karlsruhe.

Pahl, G., Beitz, W., Feldhusen, J. and Grote, K.-H. (2007), Engineering Design: A Systematic Approach, Springer, London. https://doi.org/10.1007/978-1-84628-319-2

Vetter, G. (1995), Leak-free pumps and compressors, Elsevier Science, Oxford, UK.

Ziegleder, G., Böhme, B. and Rohm, H. (2005), "Haltbarkeit von Pralinen - One-Shot-Verfahren und konventionelles Schleudern im Vergleich", Süsswaren, Vol. 50 No. 7-8, pp. 14-16.

Jean-Paul Beetz, M.Sc.

Technische Universität Darmstadt, Produktentwicklung und Maschinenelemente

Otto-Berndt-Straße 2, 64287 Darmstadt, Germany

Email: beetz@pmd.tu-darmstadt.de 